\title{
Homogenization and Scattering Analysis of Second-Order Nonlinear Metasurfaces
}

\section{K. Achouri, G. D. Bernasconi, J. Butet and O. J. F. Martin}

École Polytechnique Fédérale de Lausanne, Nanophotonics and Metrology Laboratory, Route Cantonale, 1015 Lausanne, Switzerland, karim.achouri@epfl.ch,

\begin{abstract}
We present an extensive discussion on the homogenization and scattering analysis of second-order nonlinear metasurfaces. We use the generalized sheet transition conditions (GSTCs) in the frequency-domain to model the electromagnetic responses of nonlinear metasurfaces. We present the general second-harmonic scattering relations, in the undepleted pump regime approximation, and the resulting reflectionless, transmissionless and asymmetric reflection and transmission conditions. Finally, to clarify certain misconceptions, we also discuss the concept of nonreciprocal scattering in nonlinear optics.
\end{abstract}

\section{INTRODUCTION}

Nonlinear metasurfaces have recently emerged as a new paradigm shift in the realm of nonlinear optics and have consequently attracted much attention [1,2]. In order to fully exploit their potential in controlling electromagnetic fields, one needs a general characterization of their scattering properties. This conference paper aims at presenting our most recent work pertaining to the homogenization and scattering analysis of second-order nonlinear metasurfaces [3]. This work extends our previous developments related to the electromagnetic theory of linear and nonlinear metasurfaces [4-7]. Additionally, it completes other recent works, which also cover the topics of second-order nonlinear metamaterial structures and metasurfaces [8-12].

\section{Modeling OF Second-Order Nonlinear Metasurfaces}

In the frequency-domain, assuming an undepleted pump regime and considering only the generation of secondharmonic (SH) light, the generalized sheet transition conditions (GSTCs) applied to a second-order nonlinear metasurface lying in the $x y$-plane at $z=0$ read

$$
\begin{gathered}
\boldsymbol{z} \times \Delta \boldsymbol{H}^{2 \omega}=j 2 \omega \boldsymbol{P}^{2 \omega}-\boldsymbol{z} \times \nabla M_{z}^{2 \omega}, \\
\boldsymbol{z} \times \Delta \boldsymbol{E}^{2 \omega}=-j 2 \omega \mu_{0} \boldsymbol{M}^{2 \omega}-\frac{1}{\epsilon_{0}} \boldsymbol{z} \times \nabla P_{z}^{2 \omega},
\end{gathered}
$$

where $\Delta \boldsymbol{E}^{2 \omega}$ and $\Delta \boldsymbol{H}^{2 \omega}$ represent the difference of the SH fields between both sides of the metasurface, and $\boldsymbol{P}^{2 \omega}$ and $\boldsymbol{M}^{2 \omega}$ are the effective electric and magnetic polarization densities, respectively. For a nonlinear metasurface, these polarizations may be split into linear and nonlinear contributions as $\boldsymbol{P}^{2 \omega}=\boldsymbol{P}_{\operatorname{lin}}^{2 \omega}+\boldsymbol{P}_{\mathrm{nl}}^{2 \omega}$ and $\boldsymbol{M}^{2 \omega}=$ $\boldsymbol{M}_{\mathrm{lin}}^{2 \omega}+\boldsymbol{M}_{\mathrm{nl}}^{2 \omega}$. The linear polarizations model how the SH fields interact with the metasurface and are generally given in terms of the following bianisotropic susceptibility tensors [13]

$$
\begin{gathered}
\boldsymbol{P}_{\mathrm{lin}}^{2 \omega}=\epsilon_{0} \overline{\bar{\chi}}_{\mathrm{ee}} \cdot \boldsymbol{E}_{\mathrm{av}}^{2 \omega}+\epsilon_{0} \eta_{0} \overline{\bar{\chi}}_{\mathrm{em}} \cdot \boldsymbol{H}_{\mathrm{av}}^{2 \omega}, \\
\boldsymbol{M}_{\mathrm{lin}}^{2 \omega}=\overline{\bar{\chi}}_{\mathrm{mm}} \cdot \boldsymbol{H}_{\mathrm{av}}^{2 \omega}+\frac{1}{\eta_{0}} \overline{\bar{\chi}}_{\mathrm{me}} \cdot \boldsymbol{E}_{\mathrm{av}}^{2 \omega},
\end{gathered}
$$

where the subscript "av" refers to the arithmetic average of the SH fields on both sides of the metasurface. Similarly, the nonlinear polarizations model how the SH fields are generated by the interactions of the pump fields with the metasurface nonlinear susceptibility tensors. These nonlinear polarizations may generally be expressed as

$$
\boldsymbol{P}_{\mathrm{nl}}^{2 \omega}=\frac{1}{2} \epsilon_{0}\left(\overline{\bar{\chi}}_{\mathrm{eee}}: \boldsymbol{E}_{\mathrm{av}}^{\omega} \boldsymbol{E}_{\mathrm{av}}^{\omega}+\eta_{0} \overline{\bar{\chi}}_{\mathrm{eem}}: \boldsymbol{E}_{\mathrm{av}}^{\omega} \boldsymbol{H}_{\mathrm{av}}^{\omega}+\eta_{0}^{2} \overline{\bar{\chi}}_{\mathrm{emm}}: \boldsymbol{H}_{\mathrm{av}}^{\omega} \boldsymbol{H}_{\mathrm{av}}^{\omega}\right),
$$




$$
\boldsymbol{M}_{\mathrm{nl}}^{2 \omega}=\frac{1}{2}\left(\eta_{0} \overline{\bar{\chi}}_{\mathrm{mmm}}: \boldsymbol{H}_{\mathrm{av}}^{\omega} \boldsymbol{H}_{\mathrm{av}}^{\omega}+\overline{\bar{\chi}}_{\mathrm{mem}}: \boldsymbol{E}_{\mathrm{av}}^{\omega} \boldsymbol{H}_{\mathrm{av}}^{\omega}+\frac{1}{\eta_{0}} \overline{\bar{\chi}}_{\mathrm{mee}}: \boldsymbol{E}_{\mathrm{av}}^{\omega} \boldsymbol{E}_{\mathrm{av}}^{\omega}\right) .
$$

Using (1) along with (2) and (3), one may either homogenize a nonlinear metasurface, i.e. find its effective susceptibility components in terms of known (measured or simulated) fields, or compute the fields scattered by a metasurface with known susceptibilities [3]. The operation of homogenization is already discussed in the literature [8-12]. As a consequence, we next concentrate our attention on the scattering properties of these structures. Note that here, we only provide the main results of this scattering analysis while the details of the derivations may be found in [3]. For this analysis, we assume that the pump is normally incident on a spatially uniform nonlinear metasurface. The general expressions for the backward $(z<0)$ and forward $(z>0) \mathrm{SH}$ scattered fields are found by solving (1) with (2) and (3), which gives

$$
\begin{aligned}
& \boldsymbol{E}_{\mathrm{bw}}^{2 \omega}=\frac{1}{\epsilon_{0}} \overline{\overline{\mathrm{C}}}_{1} \cdot \boldsymbol{P}_{\mathrm{nl}}^{2 \omega}+\eta_{0} \overline{\overline{\mathrm{C}}}_{2} \cdot \boldsymbol{M}_{\mathrm{nl}}^{2 \omega}, \\
& \boldsymbol{E}_{\mathrm{fw}}^{2 \omega}=\frac{1}{\epsilon_{0}} \overline{\overline{\mathrm{C}}}_{3} \cdot \boldsymbol{P}_{\mathrm{nl}}^{2 \omega}-\eta_{0} \overline{\overline{\mathrm{C}}}_{4} \cdot \boldsymbol{M}_{\mathrm{nl}}^{2 \omega},
\end{aligned}
$$

where the tensors $\overline{\overline{\mathrm{C}}}$ are $^{1}$

$$
\begin{aligned}
& \overline{\overline{\mathrm{C}}}_{1}=-j 2 \overline{\overline{\mathrm{A}}} \cdot\left(\frac{c_{0}}{\omega} \overline{\overline{\mathbf{J}}}+j \overline{\bar{\chi}}_{\mathrm{mm}} \cdot \overline{\overline{\mathbf{J}}}+j \overline{\bar{\chi}}_{\mathrm{me}}\right) \cdot\left(\frac{c_{0}}{\omega} \overline{\overline{\mathbf{I}}}+j \overline{\bar{\chi}}_{\mathrm{ee}}+j \overline{\bar{\chi}}_{\mathrm{em}} \cdot \overline{\overline{\mathbf{J}}}\right)^{-1}, \\
& \overline{\overline{\mathrm{C}}}_{2}=-j 2 \overline{\overline{\mathrm{A}}} \cdot \overline{\overline{\mathbf{J}}}, \\
& \overline{\overline{\mathrm{C}}}_{3}=j 2\left(\frac{c_{0}}{\omega} \overline{\overline{\mathrm{I}}}+j \overline{\bar{\chi}}_{\mathrm{ee}}+j \overline{\bar{\chi}}_{\mathrm{em}} \cdot \overline{\overline{\mathbf{J}}}\right)^{-1} \cdot\left[\left(\frac{c_{0}}{\omega} \overline{\overline{\mathbf{I}}}+j \overline{\bar{\chi}}_{\mathrm{ee}}-j \overline{\bar{\chi}}_{\mathrm{em}} \cdot \overline{\overline{\mathbf{J}}}\right) \cdot \overline{\overline{\mathrm{A}}}\right. \\
& \left.\cdot\left(\frac{c_{0}}{\omega} \overline{\overline{\mathbf{J}}}+j \overline{\bar{\chi}}_{\mathrm{mm}} \cdot \overline{\overline{\mathbf{J}}}+j \overline{\bar{\chi}}_{\mathrm{me}}\right) \cdot\left(\frac{c_{0}}{\omega} \overline{\overline{\mathrm{I}}}+j \overline{\bar{\chi}}_{\mathrm{ee}}+j \overline{\bar{\chi}}_{\mathrm{em}} \cdot \overline{\overline{\mathbf{J}}}\right)^{-1}-\overline{\overline{\mathrm{I}}}\right] \text {, } \\
& \overline{\overline{\mathbf{C}}}_{4}=-j 2\left(\frac{c_{0}}{\omega} \overline{\overline{\mathbf{I}}}+j \overline{\bar{\chi}}_{\mathrm{ee}}+j \overline{\bar{\chi}}_{\mathrm{em}} \cdot \overline{\overline{\mathbf{J}}}\right)^{-1} \cdot\left(\frac{c_{0}}{\omega} \overline{\overline{\mathbf{I}}}+j \overline{\bar{\chi}}_{\mathrm{ee}}-j \overline{\bar{\chi}}_{\mathrm{em}} \cdot \overline{\overline{\mathbf{J}}}\right) \cdot \overline{\overline{\mathrm{A}}} \cdot \overline{\overline{\mathbf{J}}},
\end{aligned}
$$

with the matrix $\overline{\overline{\mathrm{A}}}$ given by

$\overline{\overline{\mathrm{A}}}=\left[\left(\frac{c_{0}}{\omega} \overline{\overline{\mathbf{J}}}+j \overline{\bar{\chi}}_{\mathrm{mm}} \cdot \overline{\overline{\mathbf{J}}}-j \overline{\bar{\chi}}_{\mathrm{me}}\right)+\left(\frac{c_{0}}{\omega} \overline{\mathbf{J}}+j \overline{\bar{\chi}}_{\mathrm{mm}} \cdot \overline{\overline{\mathbf{J}}}_{+j} \overline{\bar{\chi}}_{\mathrm{me}}\right) \cdot\left(\frac{c_{0}}{\omega} \overline{\overline{\mathbf{I}}}+j \overline{\bar{\chi}}_{\mathrm{ee}}+j \overline{\bar{\chi}}_{\mathrm{em}} \cdot \overline{\overline{\mathbf{J}}}\right)^{-1} \cdot\left(\frac{c_{0}}{\omega} \overline{\overline{\mathbf{I}}}+j \overline{\bar{\chi}}_{\mathrm{ee}}-j \overline{\bar{\chi}}_{\mathrm{em}} \cdot \overline{\overline{\mathbf{J}}}\right)\right]^{-1}$

and where

$$
\overline{\overline{\mathrm{I}}}=\left(\begin{array}{ll}
1 & 0 \\
0 & 1
\end{array}\right) \quad \text { and } \quad \overline{\overline{\mathrm{J}}}=\left(\begin{array}{cc}
0 & -1 \\
1 & 0
\end{array}\right) .
$$

The procedure to compute the SH scattered fields is thus as follows: 1) compute the linear fields scattered by the interactions of the pump with the metasurface using conventional methods applying to linear metasurfaces $[4,6]$, 2) use these fields to define the nonlinear polarizations in (3), 3) the SH scattered fields are then found using (4) with (5) and (6). An important consideration is that the nonlinear polarizations in (3) are not the same for a pump propagating in the forward or backward direction. This has particularly interesting implications since it notably allows one to generate unidirectional SH fields [7-12].

Using relations (4), we have derived the general reflectionless and transmissionless conditions and corresponding asymmetric reflection and transmission conditions. For conciseness, these conditions are not provided here but will be presented at the conference and may be found in [3]. These conditions notably show that a nonlinear metasurface cannot be reflectionless from both sides as it is the case for conventional linear metasurfaces. This implies that the SH scattering is generally dependent on the direction of propagation of the pump. This peculiar behavior may a priori corresponds to a nonreciprocal effect. However, we will show that this is not the case and that it is rather a consequence of the asymmetric scattering produced by some of the nonlinear susceptibilities in (3).

\footnotetext{
${ }^{1}$ In these tensors, the susceptibilities have to be evaluated at the frequency $2 \omega$.
} 


\section{CONCLUSION}

This work presents the homogenization and scattering analysis of second-order nonlinear metasurfaces in the undepleted pump regime approximation. We use the GSTCs in the frequency-domain to develop a general model of this kind of metasurfaces. This model is then used to derive the general SH scattering relations and the corresponding scattering properties, which leads to the reflectionless and transmissionless conditions.

At the conference, we will also discuss and compare the differences between the SH asymmetric scattering properties and the nonreciprocal behavior of these nonlinear metasurfaces. Additionally, we will present numerical simulations of plasmonic nonlinear scattering particles in order to illustrate the homogenization and scattering behavior of second-order nonlinear metasurfaces.

\section{REFERENCES}

[1] A. Krasnok, M. Tymchenko, and A. Al, "Nonlinear metasurfaces: a paradigm shift in nonlinear optics," Materials Today, 2017.

[2] J. Lee, M. Tymchenko, C. Argyropoulos, P.-Y. Chen, F. Lu, F. Demmerle, G. Boehm, M.-C. Amann, A. Alù, and M. A. Belkin, "Giant nonlinear response from plasmonic metasurfaces coupled to intersubband transitions," Nature, vol. 511, pp. $65 \mathrm{EP}-$, Jul 2014.

[3] K. Achouri, G. D. Bernasconi, J. Butet, and O. J. F. Martin, "Homogenization and scattering analysis of second-harmonic generation in nonlinear metasurfaces," arXiv preprint arXiv:1802.10477, 2018.

[4] K. Achouri, M. A. Salem, and C. Caloz, "General metasurface synthesis based on susceptibility tensors," IEEE Trans. Antennas Propag., vol. 63, no. 7, pp. 2977-2991, Jul. 2015.

[5] K. Achouri, B. A. Khan, S. Gupta, G. Lavigne, M. A. Salem, and C. Caloz, "Synthesis of electromagnetic metasurfaces: principles and illustrations," EPJ Applied Metamaterials, vol. 2, p. 12, 2015.

[6] K. Achouri and C. Caloz, "Design, concepts and applications of electromagnetic metasurfaces," arXiv preprint arXiv:1712.00618, 2017.

[7] K. Achouri, Y. Vahabzadeh, and C. Caloz, "Mathematical synthesis and analysis of a second-order magneto-electrically nonlinear metasurface," Opt. Express, vol. 25, no. 16, pp. 19013-19022, Aug 2017.

[8] A. Rose, S. Larouche, E. Poutrina, and D. R. Smith, "Nonlinear magnetoelectric metamaterials: Analysis and homogenization via a microscopic coupled-mode theory," Phys. Rev. A, vol. 86, p. 033816, Sep 2012.

[9] A. Rose, D. Huang, and D. R. Smith, "Nonlinear interference and unidirectional wave mixing in metamaterials," Phys. Rev. Lett., vol. 110, p. 063901, Feb 2013.

[10] E. Poutrina and A. Urbas, "Multipole analysis of unidirectional light scattering from plasmonic dimers," Journal of Optics, vol. 16, no. 11, p. 114005, 2014.

[11] E. Poutrina and A. Urbas, "Multipolar interference for non-reciprocal nonlinear generation," Scientific Reports, vol. 6, pp. 25113 EP -, Apr 2016.

[12] X. Liu, S. Larouche, and D. R. Smith, "Homogenized description and retrieval method of nonlinear metasurfaces," Optics Communications, vol. 410, pp. 53 - 69, 2018.

[13] A. H. Sihvola, A. J. Viitanen, I. V. Lindell, and S. A. Tretyakov, Electromagnetic waves in chiral and bi-isotropic media, ser. The Artech House Antenna Library. Artech House, 1994. 